\title{
Optimal invariance via receding horizon control
}

\author{
Lars Grüne
}

\begin{abstract}
We analyze the performance of receding horizon controllers for the problem of keeping the state of a system within a given admissible set while minimizing an averaged functional. This problem does not satisfy the usual conditions needed for the analysis of receding horizon schemes. We give conditions under which approximate optimal performance can be guaranteed without imposing terminal constraints and illustrate our results by means of two numerical examples.
\end{abstract}

\section{INTRODUCTION}

In this paper we investigate a receding horizon $(\mathrm{RH})$ approach for the following feedback control problem: keep the state of a (possibly nonlinear) discrete time system within an admissible set $\mathbb{X}$ while minimizing an averaged performance criterion. For the solution of the problem we follow the usual RH (or model predictive control) paradigm. In each time step for the current state we optimize the averaged functional subject to the state constraints over a finite horizon and apply the first element of the resulting optimal control sequence as a feedback value for the next time step.

Most results for (linear and nonlinear) RH control schemes are developed for optimal control problems in which the stage cost penalizes the distance to a desired equilibrium or a more general reference solution. Stability and performance results can be obtained under additional stabilizing terminal constraints (see, e.g., [9], [11] or [7, Chapter 5]) or without such constraints (see, e.g., [5], [6], [8] or [7, Chapter 6]). These results require the stage cost to be positive definite in the state or use a more general detectability condition as in [5] or an input/output-to-state stability condition as in [11, Section 2.7 and the references therein].

Here we consider optimal control problems which do not satisfy these conditions. For such problems, which arise, e.g., if the stage cost models economic costs instead of penalizing a distance to a desired reference, recently a two stage procedure was analyzed in [1], [4], [2]. In this procedure, one first determines an optimal equilibrium or periodic orbit for the original problem and then uses this solution as a terminal constraint for the RH scheme. With this approach the infinite horizon average performance of the RH controller equals that of the original problem and under additional conditions also convergence of the $\mathrm{RH}$ closed loop solution to the optimal equilibrium or periodic orbit can be ensured. One of the valuable insights of these references is that an averaged functional is the right object for obtaining such results. Consequently, in this paper we also

Chair of Applied Mathematics, University of Bayreuth, 95440 Bayreuth, Germany, lars.gruene@uni-bayreuth. de. Research supported by DFG Grant Gr1569/12-2 within the Priority Research Program 1305. use this performance criterion. In contrast to these references, however, we do not impose any terminal constraints. Instead, we derive conditions under which RH controllers are able to yield (approximately) optimal performance without including a priori information about optimal solutions in the $\mathrm{RH}$ formulation.

The paper is organized as follows. After formulating the problem and premilinary results in Section II we discuss a numerical example for a simple 1d system in Section III. This example on the one hand shows that convergence can be expected and on the other hand helps to identify suitable conditions for the main results of the paper which can be found in Section IV. Here we first present a general theorem which yields an upper bound for both finite and infinite horizon average performance of the RH closed loop and then formulate a corollary giving sufficient conditions in terms of the system dynamics which in particular apply to our first example. In order to make the main arguments more transparent these results are formulated for the case of an optimal equilibrium; an extension to optimal periodic solutions is discussed by means of an example in Section V. Finally, Section VI concludes the paper.

\section{PROBLEM FORMULATION AND PRELIMINARIES}

We consider discrete time control systems with state $x \in$ $X$ and control values $u \in U$, where $X$ and $U$ are subsets of normed spaces with norms denoted by $\|\cdot\|$. The control system under consideration is given by

$$
x(k+1)=f(x(k), u(k))
$$

with $f: X \times U \rightarrow X$. For a given control sequence $u=$ $(u(0), \ldots, u(K-1)) \in U^{K}$ or $u=(u(0), u(1), \ldots) \in U^{\infty}$, by $x_{u}(k, x)$ we denote the solution of (1) with initial value $x=x_{u}(0, x) \in X$.

For a given admissible set $\mathbb{X} \subset X$ and an initial value $x \in X$ we call the control sequences $u \in U^{K}$ satisfying

$$
x_{u}(k, x) \in \mathbb{X} \quad \text { for all } k=0, \ldots, K
$$

admissible. The set of all admissible control sequences is denoted by $\mathbb{U}^{K}(x)$. Similarly, we define the set $\mathbb{U}^{\infty}(x)$ of admissible control sequences of infinite length. Since the emphasis of the analysis in this paper is on optimality rather than on feasibility, for simplicity of exposition we assume $\mathbb{U}^{\infty}(x) \neq \emptyset$ for all $x \in \mathbb{X}$, i.e., that for each initial value $x \in \mathbb{X}$ we can find a trajectory staying inside $\mathbb{X}$ for all future times. This condition may be relaxed if desired, using, e.g., the techniques from [7, Sections 8.2-8.3] or [10]. 
Given a feedback map $\mu: X \rightarrow U$, we denote the solutions of the closed loop system

$$
x(k+1)=f(x(k), \mu(x(k)))
$$

by $x_{\mu}(k)$ or by $x_{\mu}(k, x)$ if we want to emphasize the dependence on the initial value $x=x_{\mu}(0)$. We say that a feedback law $\mu$ is admissible if it renders the admissible set $\mathbb{X}$ (forward) invariant, i.e., if $f(x, \mu(x)) \in \mathbb{X}$ holds for all $x \in \mathbb{X}$. Note that $\mathbb{U}^{\infty}(x) \neq \emptyset$ for all $x \in \mathbb{X}$ immediately implies that such a feedback law exists.

Our goal is now to find an admissible feedback controller which yields trajectories with minimal average cost. To this end, for a given running cost $\ell: X \times U \rightarrow \mathbb{R}$ we define the following averaged functionals and optimal value functions.

$$
\begin{aligned}
J_{N}(x, u) & :=\frac{1}{N} \sum_{k=0}^{N-1} \ell\left(x_{u}(k, x), u(k)\right) \\
J_{\infty}(x, u) & :=\limsup _{N \rightarrow \infty} J_{N}(x, u) \\
V_{N}(x) & :=\inf _{u \in \mathbb{U}^{N}(x)} J_{N}(x, u) \\
V_{\infty}(x) & :=\inf _{u \in \mathbb{U}^{\infty}(x)} J_{\infty}(x, u)
\end{aligned}
$$

Here we assume that $\ell$ is bounded from below on $\mathbb{X}$, i.e., that $\ell_{\min }:=\inf _{x \in \mathbb{X}, u \in U} \ell(x, u)$ is finite. Without loss of generality we may assume $\ell_{\min }=0$; otherwise we may replace $\ell$ by $\ell-\ell_{\min }$. This assumption immediately yields that all functionals are nonnegative for each $x \in \mathbb{X}$ and all admissible control sequences. In order to simplify the exposition in what follows, we assume that (not necessarily unique) optimal control sequences for $J_{N}$ exist, i.e., that for each $x \in \mathbb{X}$ and each $N \in \mathbb{N}$ there exists $u_{N, x}^{o p t} \in \mathbb{U}^{N}(x)$ satisfying

$$
V_{N}(x)=J_{N}\left(x, u_{N, x}^{o p t}\right) .
$$

Similarly to the open loop functionals, we can define the average cost of the closed loop solution for any feedback law $\mu$ by

$$
\begin{aligned}
J_{K}(x, \mu) & =\frac{1}{K} \sum_{k=0}^{K-1} \ell\left(x_{\mu}(k, x), \mu\left(x_{\mu}(k, x)\right)\right) \\
J_{\infty}(x, \mu) & =\limsup _{K \rightarrow \infty} J_{K}(x, \mu) .
\end{aligned}
$$

In order to find a feedback $\mu$ we will apply a receding horizon (RH) control scheme, also known as model predictive control. This method consists of solving the open loop optimization problem of minimizing $J_{N}(x, u)$ for some given optimization horizon $N \in \mathbb{N}$ and then defining the feedback law $\mu_{N}$ as the first element of the corresponding optimal control sequence, i.e.,

$$
\mu_{N}(x)=u_{N, x}^{o p t}(0) .
$$

We end this section by introducing some basic notation and preliminary results. As usual, with $\mathcal{K}_{\infty}$ we denote the set of continuous functions $\alpha: \mathbb{R}_{0}^{+} \rightarrow \mathbb{R}_{0}^{+}$which are strictly increasing and unbounded with $\alpha(0)=0$. With $\mathcal{L}_{\mathbb{N}}$ we denote the set of functions $\delta: \mathbb{N} \rightarrow \mathbb{R}^{+}$which are (not necessarily strictly) decreasing with $\lim _{k \rightarrow \infty} \delta(k)=0$.

In our analysis we will make extensive use of the dynamic programming principle, cf. [3]. The form of this principle we will need here states that for the optimal control sequence $u_{N, x}^{o p t}$ for the problem with finite horizon $N$ and each $K \in$ $\{1, \ldots, N-1\}$ the equality

$$
\begin{aligned}
V_{N}(x)= & \frac{1}{N} \sum_{k=0}^{K-1} \ell\left(x_{u_{N, x}^{o p t}}(k, x), u_{N, x}^{o p t}(k)\right) \\
& +\frac{N-K}{N} V_{N-K}\left(x_{u_{N, x}^{o p t}}(K, x)\right)
\end{aligned}
$$

holds. As a consequence, for $\mu_{N}(x)=u_{N, x}^{o p t}(0)$ we get

$$
V_{N}(x)=\frac{1}{N} \ell\left(x, \mu_{N}(x)\right)+\frac{N-1}{N} V_{N-1}\left(f\left(x, \mu_{N}(x)\right)\right) .
$$

This implies the equation

$$
\begin{gathered}
\ell\left(x, \mu_{N}(x)\right)=N V_{N}(x)-(N-1) V_{N-1}\left(f\left(x, \mu_{N}(x)\right)\right) . \\
\text { III. A MOTIVATING EXAMPLE }
\end{gathered}
$$

In order to illustrate how receding horizon control performs for the optimal invariance problem under consideration, we look at the following motivating example.

Example 1: Consider the control system

$$
x(k+1)=2 x(k)+u(k)
$$

with $X=\mathbb{R}$ and $U=[-2,2]$. The running cost $\ell$ is chosen such that the control effort is penalized quadratically, i.e., $\ell(x, u)=u^{2}$. We consider the admissible sets $\mathbb{X}=[-a, a]$ with $a=0.5$ and $a=1$. For these sets it is easily seen that an optimal way of keeping the solutions inside $\mathbb{X}$ in the infinite horizon averaged sense is to steer the system to $x^{*}=0$ in a finite number of steps $k^{*}$ and set $u(k)=0$ for $k \geq k^{*}$ which leads to $J_{\infty}(x, u)=0$. Since $\ell(x, u) \geq 0$ for all $x$ and $u$, this is the optimal value of $J_{\infty}$, i.e., $V_{\infty}(x)=0$ for all $x \in \mathbb{X}$.

This example does not satisfy the usual conditions imposed on receding horizon control schemes in the literature. Indeed, since we do not impose terminal constraints neither the techniques for stabilizing RH schemes presented, e.g., in [11] or [9] nor the techniques for economic problems from [1], [4], [2] apply. The results from [6], [8] do not apply, either, because the running cost $\ell$ is not positive definite in the state $x$. Finally, the detectability condition from [5] fails to hold for the equilibrium $x^{*}$ : this conditions requires the existence of a nonnegative function $W: \mathbb{X} \rightarrow \mathbb{R}_{0}^{+}$ satisfying $W(x) \leq \alpha_{1}(|x|)$ and $W(f(x, u))-W(x) \leq$ $-\alpha_{2}(|x|)+\gamma(\ell(x, u))$ for suitable functions $\alpha_{2}, \gamma \in \mathcal{K}_{\infty}$ and a continuous nondecreasing function $\alpha_{1}: \mathbb{R}_{0}^{+} \rightarrow \mathbb{R}_{0}^{+}$ with $\alpha_{1}(0)=0$. Assuming that such a function $W$ exists, using $u=0$ and $\ell(x, 0)=0$ implies $W(2 x)-W(x) \leq$ $-\alpha_{2}(|x|)$ which yields $W(x) \leq W(x / 2)-\alpha_{2}(|x| / 2)$ for all $x \in \mathbb{X}$ with $x \neq 0$. By iterating this inequality and using $\alpha_{2}(\cdot) \geq 0$ we get $W(x) \leq W\left(x / 2^{i}\right)-\alpha_{2}(|x| / 2)$ for all $i \in \mathbb{N}$. For $i \rightarrow \infty$ we get $W\left(x / 2^{i}\right) \leq \alpha_{1}\left(|x| / 2^{i}\right) \rightarrow 0$ 
implying $W(x) \leq-\alpha_{2}(|x| / 2)<0$ which contradicts the nonnegativity of $W$.

Nevertheless, the receding horizon feedback $\mu_{N}$ produces approximately optimal closed loop solutions. In order to illustrate this fact, we have simulated it numerically in Matlab using the fmincon optimization routine (for details see [7, Appendix A]).

Figure 1 shows the infinite horizon averaged value $J_{\infty}\left(x, \mu_{N}\right)$ for the receding horizon strategy thus obtained for different optimization horizons $N$ and the two admissible sets $\mathbb{X}=[-1,1]$ (solid) and $\mathbb{X}=[-0.5,0.5]$ (dashed). The values are plotted on a logarithmic scale and indicate that $J_{\infty}\left(x, \mu_{N}\right) \rightarrow 0$ as $N \rightarrow \infty$.

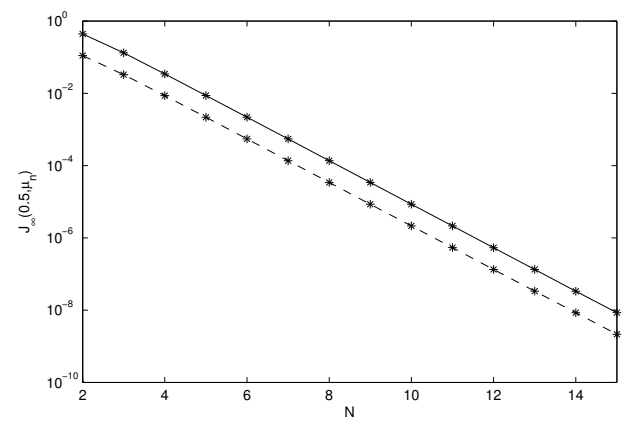

Fig. 1. $\quad J_{\infty}\left(x, \mu_{N}\right)$ for $N=2, \ldots, 15$ and $x=0.5, \mathbb{X}=[1,1]$ (solid) and $\mathbb{X}=[-0.5,0.5]$ (dashed)

We observe: for increasing optimization horizon $N$ the closed loop infinite horizon averaged values $J_{\infty}\left(x, \mu_{N}\right)$ improve and approach the optimum $V_{\infty}(x)=0$ as $N \rightarrow \infty$. On the other hand, for the larger admissible set $\mathbb{X}=[-1,1]$ the values are larger — despite the fact that the infinite horizon optimal value does not depend on the choice of $\mathbb{X}$.

Figure 2 shows the corresponding closed loop trajectories for $\mathbb{X}=[-0.5,0.5]$ with optimization horizon $N=5$ (solid) and $N=10$ (dashed).

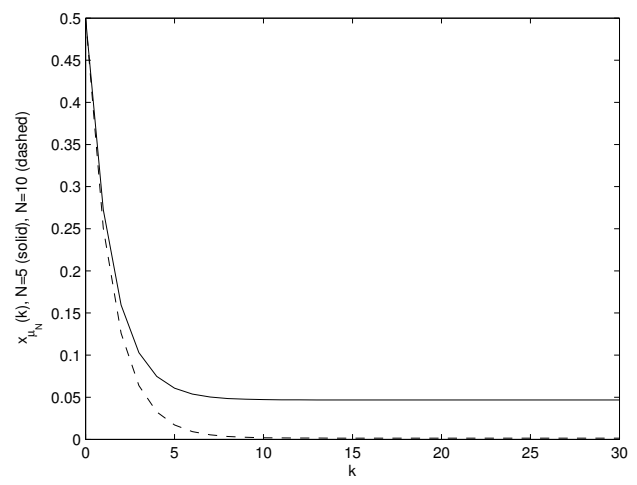

Fig. 2. $\quad x_{\mu_{N}}(k, x)$ for $N=5$ (solid) and $N=10$ (dashed), both for $x=0.5$ and $\mathbb{X}=[-0.5,0.5]$

It is interesting to compare the closed loop trajectories with the optimal open loop trajectories in each step of the scheme, as illustrated in Figure 3 for $\mathbb{X}=[-1,1]$ and $N=5$.
While the closed loop trajectory approaches a neighborhood of $x^{*}=0$, the optimal open loop trajectories tend towards the upper boundary $x=1$ of the admissible set $\mathbb{X}=[-1,1]$.

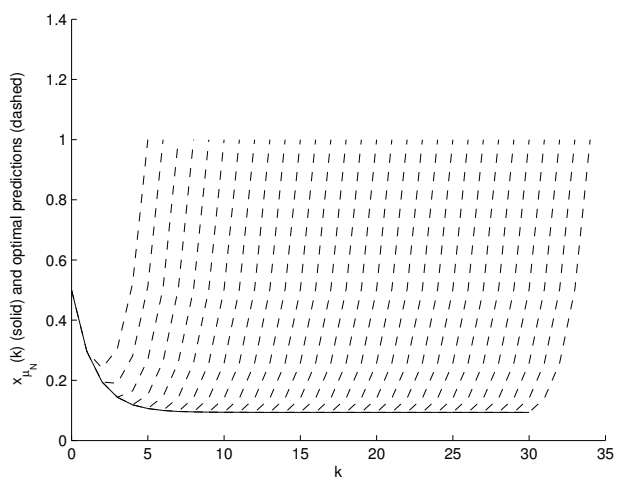

Fig. 3. Optimal predictions $x_{u}\left(k, x_{\mu_{N}}(k)\right)$ (dashed) within the receding horizon optimization for $N=5, x=0.5$ and $\mathbb{X}=[-1,1]$

\section{MAIN RESULT}

Our goal now is to investigate the dependence of $J_{\infty}\left(x, \mu_{N}\right)$ on $N$. The following theorem gives an upper bound for this value. Its proof uses the classical RH proof technique to prolong a suitable control sequence of length $N$ in order to obtain a sequence of length $N+1$ for which the difference between $J_{N+1}$ and $V_{N}$ can be estimated. However, since we have seen in Figure 3 that the optimal trajectories for the finite horizon problem end up at the boundary of the admissible set, using optimal control sequences for $J_{N}$ for this purpose will in general not lead to a good estimate. For instance, in the case of Example 1 we have $x_{u}(N)=1$ (cf. Figure 3) and would thus have to use $u(N)=-2$ in order to obtain feasibility, i.e., to guarantee $x_{u}(N+1)=f\left(x_{u}(N), u(N)\right) \in \mathbb{X}$. This leads to $\ell\left(x_{u}(N), u(N)\right)=4$ which is much larger than the minimal value $\min _{x, u} \ell(x, u)=0$ of $\ell$. For this reason, in the assumptions of the following theorem we use approximate optimal control sequences $u_{N, x}$ instead of optimal ones.

Theorem 2: Assume there are $N_{0}>0$ and $\delta_{1}, \delta_{2} \in \mathcal{L}_{\mathbb{N}}$ such that for each $x \in \mathbb{X}$ and $N \geq N_{0}$ there exists a control sequence $u_{N, x} \in \mathbb{U}^{N+1}$ satisfying the following conditions.

(i) The inequality

$$
J_{N}\left(x, u_{N, x}\right) \leq V_{N}(x)+\delta_{1}(N) / N
$$

holds, i.e., $u_{N, x}$ is approximately optimal for $J_{N}$ with error $\delta_{1}(N) / N$.

(ii) There exists $\ell_{0} \in \mathbb{R}$ such that for all $x \in \mathbb{X}$

$$
\ell\left(x_{u_{N, x}}(N, x), u_{N, x}(N)\right) \leq \ell_{0}+\delta_{2}(N)
$$

holds.

Then the inequalities

$$
\begin{aligned}
J_{K}\left(x, \mu_{N}\right) \leq & \frac{N}{K} V_{N}(x)+\ell_{0}-\frac{N-1}{K} V_{N-1}(k) \\
& +\delta_{1}(N-1)+\delta_{2}(N-1)
\end{aligned}
$$


and

$$
J_{\infty}\left(x, \mu_{N}\right) \leq \ell_{0}+\delta_{1}(N-1)+\delta_{2}(N-1)
$$

hold for all $x \in \mathbb{X}$, all $N \geq N_{0}+1$ and all $K \in \mathbb{N}$.

Proof: Fix $x \in \mathbb{X}$ and $N \geq N_{0}+1$. Abbreviating $x(k)=x_{\mu_{N}}(k, x)$, from (3) for any $k \geq 0$ we get

$$
\begin{aligned}
& \frac{1}{K} \ell\left(x(k), \mu_{N}(x(k))\right) \\
& \quad=\frac{N}{K} V_{N}(x(k))-\frac{N-1}{K} V_{N-1}(x(k+1)) .
\end{aligned}
$$

Summing up for $k=0, \ldots, K-1$ then yields

$$
\begin{aligned}
J_{K}\left(x, \mu_{N}\right) \\
=\frac{1}{K} \sum_{k=0}^{K-1} \ell\left(x(k), \mu_{N}(x(k))\right) \\
=\sum_{k=0}^{K-1}\left(\frac{N}{K} V_{N}(x(k))-\frac{N-1}{K} V_{N-1}(x(k+1))\right) \\
=\frac{N}{K} V_{N}(x(0))-\frac{N-1}{K} V_{N-1}(x(K)) \\
\quad+\frac{1}{K} \sum_{k=1}^{K-1}\left(N V_{N}(x(k))-(N-1) V_{N-1}(x(k))\right) .
\end{aligned}
$$

Now we investigate the terms in (6). From (i) applied with $N-1$ in place of $N$ and $x=x(k)$ we get the inequality

$$
\begin{aligned}
(N-1) V_{N-1}(x(k)) \geq & (N-1) J_{N-1}\left(x(k), u_{N-1, x(k)}\right) \\
& -\delta_{1}(N-1) .
\end{aligned}
$$

Furthermore, by optimality of $V_{N}$ we get

$$
V_{N}(x(k)) \leq J_{N}\left(x(k), u_{N-1, x(k)}\right) .
$$

Combining these inequalities, using the definition of $J_{N}$ and (ii), for the summands of (6) we get

$$
\begin{aligned}
& N V_{N}(x(k))-(N-1) V_{N-1}(x(k)) \\
& \leq N J_{N}\left(x(k), u_{N-1, x(k)}\right) \\
& \quad-(N-1) J_{N-1}\left(x(k), u_{N-1, x(k)}\right)+\delta_{1}(N-1) \\
& =\ell\left(x_{u_{N-1, x(k)}}(N-1, x(k)), u_{N-1, x(k)}(N-1)\right) \\
& \quad+\delta_{1}(N-1) \\
& \leq \ell_{0}+\delta_{2}(N-1)+\delta_{1}(N-1) .
\end{aligned}
$$

Inserting these inequalities into (6) yields

$$
\begin{aligned}
J_{K}\left(x, \mu_{N}\right) \leq & \frac{N}{K} V_{N}(x)-\frac{N-1}{K} V_{N-1}(x(K)) \\
& +\ell_{0}+\delta_{2}(N-1)+\delta_{1}(N-1),
\end{aligned}
$$

i.e., (4). Inequality (5) follows from (4) by letting $K \rightarrow \infty$ since $V_{N-1}$ is nonnegative.

The subtle point in Theorem 2 is that the approximation error in (i) must tend to 0 faster than $1 / N$. The following corollary gives conditions on the dynamics of the system on $\mathbb{X}$ under which we can construct such trajectories in presence of an optimal equilibrium. As we will see after the proof, these conditions in particular apply to Example 1.
Corollary 3: Assume that $\mathbb{X}$ is bounded and that there exists $x^{*} \in \mathbb{X}$ and $u^{*} \in \mathbb{U}$ such that $f\left(x^{*}, u^{*}\right)=x^{*}$ and $\ell_{0}:=\ell\left(x^{*}, u^{*}\right)=\min _{(x, u) \in \mathbb{X} \times \mathbb{U}} \ell(x, u)$ holds. Assume furthermore that the following two properties hold.

(a) There exists $R \in \mathbb{N}$ and $\alpha \in \mathcal{K}_{\infty}$ such that for each $x \in \mathbb{X}$ there exists $u_{x} \in \mathbb{U}^{R}(x)$ with $x_{u_{x}}\left(k_{x}, x\right)=x^{*}$ for some $k_{x} \leq R$ and

$$
\ell\left(x_{u_{x}}(k, x), u_{x}(k)\right) \leq \ell_{0}+\alpha\left(\left\|x-x^{*}\right\|\right)
$$

for all $k=0, \ldots, k_{x}-1$.

(b) There exists $\gamma, \delta \in \mathcal{L}_{\mathbb{N}}$ and $N_{0}>0$ such that for all $N \geq N^{\prime} \geq N_{0}$, each $x \in \mathbb{X}$ and each trajectory $x_{u}(k, x)$ satisfying $\left\|x_{u}(k, x)-x^{*}\right\| \geq \delta\left(N^{\prime}\right)$ and $x_{u}(k, x) \in \mathbb{X}$ for all $k=0, \ldots, N$ the inequality

$$
J_{N}(x, u) \geq \ell_{0}+\gamma\left(N^{\prime}\right)
$$

holds.

Then $V_{\infty}(x)=\ell_{0}$ holds for all $x \in \mathbb{X}$ and there exists $\varepsilon \in \mathcal{L}_{\mathbb{N}}$ and $\widetilde{N}_{0} \in \mathbb{N}$ such that the inequality

$$
J_{\infty}\left(x, \mu_{N}\right) \leq \ell_{0}+\varepsilon(N-1)=V_{\infty}(x)+\varepsilon(N-1)
$$

holds for all $x \in \mathbb{X}$ and all $N \geq \widetilde{N}_{0}+1$.

Proof: We first derive a priori bounds on $V_{N}$ and $V_{\infty}$ for $x \in \mathbb{X}$. From the assumptions on $\ell$ it immediately follows that $V_{N}(x) \geq \ell_{0}$ and $V_{\infty}(x) \geq \ell_{0}$ for all $x \in \mathbb{X}$ and $N \in \mathbb{N}$. In order to derive upper bounds for $V_{N}$ and $V_{\infty}$ consider $x \in \mathbb{X}$ and the control sequence $\tilde{u}_{x} \in \mathbb{U}^{\infty}(x)$ defined by

$$
\tilde{u}_{x}(k):= \begin{cases}u_{x}(k), & k=0, \ldots, k_{x}-1 \\ u^{*}, & k \geq k_{x}\end{cases}
$$

with $u_{x}$ and $k_{x}$ from (a). Then it follows that $x_{\tilde{u}_{x}}(k, x)=x^{*}$ and $\ell\left(x_{\tilde{u}_{x}}(k, x), \tilde{u}_{x}\right)=\ell_{0}$ for all $k \geq k_{x}$ and

$$
\ell\left(x_{\tilde{u}_{x}}(k, x), \tilde{u}_{x}\right) \leq \ell_{0}+\alpha\left(\left\|x-x^{*}\right\|\right)
$$

for all $k \in \mathbb{N}$. Thus we get

$$
J_{N}\left(x, \tilde{u}_{x}\right) \leq \ell_{0}+\frac{k_{x}}{N} \alpha\left(\left\|x-x^{*}\right\|\right)
$$

for all $N \in \mathbb{N}$ from which

$$
V_{N}(x) \leq \ell_{0}+\frac{k_{x}}{N} \alpha\left(\left\|x-x^{*}\right\|\right) \quad \text { and } \quad V_{\infty}(x) \leq \ell_{0}
$$

follows. In particular, this implies $V_{\infty}(x)=\ell_{0}$.

We now construct $u_{N, x}$ meeting the assumptions of Theorem 2 . Note that $\tilde{u}_{x}$ is not suitable for this purpose, because the difference between the lower bound $\ell_{0} \leq V_{N}(x)$ and the upper bound $\ell_{0}+\frac{k_{x}}{N} \alpha\left(\left\|x-x^{*}\right\|\right) \geq J_{N}\left(x, \tilde{u}_{x}\right)$ tends to 0 slower than the gap $\delta_{1}(N) / N$ allowed in Theorem 2(i). Thus, for the construction of $u_{N, x}$ we need to exploit condition (b).

In order to construct $u_{N, x}$ we define $\alpha_{\max }:=$ $\max _{x \in \mathbb{X}} \alpha\left(\left\|x-x^{*}\right\|\right)$ (which is finite since $\mathbb{X}$ is bounded) and for each $N \geq N_{0}$ we let $\eta(N) \in\{1, \ldots, N\}$ be maximal such that $\gamma(\eta(N))>R \alpha_{\max } / N$ holds. Note that $\eta$ is nondecreasing with $\eta(N) \rightarrow \infty$ as $N \rightarrow \infty$ because $R \alpha_{\max } / N$ tends to 0 monotonically as $N \rightarrow \infty$ and $\gamma \in \mathcal{L}_{\mathbb{N}}$. We choose $\widetilde{N}_{0} \in \mathbb{N}$ minimal with $\eta\left(\widetilde{N}_{0}\right) \geq N_{0}$. 
Now we define

$$
\sigma(N):=\max \{\delta(\eta(N)), \gamma(\eta(N))\} .
$$

Since $\gamma, \delta \in \mathcal{L}_{\mathbb{N}}$ and $\eta(N) \rightarrow \infty$ monotonically as $N \rightarrow \infty$ we obtain $\sigma \in \mathcal{L}_{\mathbb{N}}$.

We claim that $\sigma$ has the following property: for each $x \in \mathbb{X}$ let $u_{N, x}^{o p t}$ be an optimal control sequence for $J_{N}(x, u)$ and some $N \geq R$ and $N \geq N_{0}$. Then

$$
\left\|x_{u_{N, x}^{o p t}}\left(k_{\sigma}, x\right)-x^{*}\right\| \leq \sigma(N)
$$

for some $k_{\sigma} \in\{0, \ldots, N\}$.

In order to show (12) let $x \in \mathbb{X}$ and assume the opposite, i.e., $\left\|x_{u_{N, x}^{o p t}}(k, x)-x^{*}\right\|>\sigma(N)$ for all $k \in\{0, \ldots, N\}$. This implies $\left\|x_{u_{N, x}^{o p t}}(k, x)-x^{*}\right\|>\delta(\eta(N))$ for all $k \in$ $\{0, \ldots, N\}$. Since $N_{0} \leq \eta(N) \leq N$, (b) applies with $N^{\prime}=$ $\eta(N)$ and yields

$$
\begin{aligned}
V_{N}(x) & =J_{N}\left(x, u_{N, x}^{o p t}\right) \geq \ell_{0}+\gamma(\eta(N)) \\
& >\ell_{0}+R \alpha_{\max } / N \geq \ell_{0}+R \alpha\left(\left\|x-x^{*}\right\|\right) / N .
\end{aligned}
$$

Since $R \geq k_{x}$ holds for $k_{x}$ from (11), this inequality contradicts (11) which proves (12).

Now we construct $u_{N, x}$ by concatenating $u_{N, x}^{o p t}$ and $\tilde{u}_{x}$ from (8) for $x=x_{u_{N, x}^{o p t}}\left(k_{\sigma}, x\right)$ with $k_{\sigma}$ from (12). Abbreviating $x_{\sigma}=x_{u_{N, x}^{o p t}}\left(k_{\sigma}, x\right)$, this amounts to defining

$$
u_{N, x}(k):= \begin{cases}u_{N, x}^{o p t}(k), & k=0, \ldots, k_{\sigma}-1 \\ \tilde{u}_{x_{\sigma}}\left(k-k_{\sigma}\right), & k \geq k_{\sigma} .\end{cases}
$$

This construction implies $x_{u_{N, x}}(k, x)=x_{u_{N, x}^{o p t}}(k, x)$ for $k=$ $0, \ldots, k_{\sigma}$ and $x_{u_{N, x}}(k, x)=x_{\tilde{u}_{x_{\sigma}}}\left(k-k_{\sigma}, x_{\sigma}\right)$ for $k \geq k_{\sigma}$. Thus, using (2) in the second step, (10) and (11) in the third step and (12) and $k_{x_{\sigma}} \leq R$ in the fourth step we get

$$
\begin{aligned}
J_{N}\left(x, u_{N, x}\right) & \\
= & \frac{1}{N} \sum_{k=0}^{k_{\sigma}-1} \ell\left(x_{u_{N, x}^{\text {opt }}}(k, x), u_{N, x}^{\text {opt }}(k)\right) \\
& \quad+\frac{1}{N} \sum_{k=0}^{N-k_{\sigma}-1} \ell\left(x_{\tilde{u}_{x_{\sigma}}}\left(k, x_{\sigma}\right), \tilde{u}_{x_{\sigma}}(k)\right) \\
= & V_{N}(x)-\frac{N-k_{\sigma}}{N} V_{N-k_{\sigma}}\left(x_{\sigma}\right) \\
& \quad+\frac{N-k_{\sigma}}{N} J_{N-k_{\sigma}}\left(x_{\sigma}, \tilde{u}_{x_{\sigma}}\right) \\
\leq & V_{N}(x) \\
& +\frac{N-k_{\sigma}}{N}\left(-\ell_{0}+\ell_{0}+\frac{k_{x_{\sigma}}}{N-k_{\sigma}} \alpha\left(\left\|x_{\sigma}-x^{*}\right\|\right)\right) \\
\leq & V_{N}(x)+\frac{R}{N} \alpha(\sigma(N)) .
\end{aligned}
$$

This implies Theorem 2(i) with $\delta_{1}(N)=R \alpha(\sigma(N))$. On the other hand, since $k_{\sigma} \leq N$ we get $u_{N, x}(N)=\tilde{u}_{x_{\sigma}}\left(N-k_{\sigma}\right)$ and $x_{u_{N, x}}(N, x)=x_{\tilde{u}_{x_{\sigma}}}\left(N-k_{\sigma}, x_{\sigma}\right)$. By (9) and (12) we thus get

$$
\begin{aligned}
\ell\left(x_{u_{N, x}}(N, x), u_{N, x}(N)\right) & \leq \ell_{0}+\alpha\left(\left\|x_{\sigma}-x^{*}\right\|\right) \\
& \leq \ell_{0}+\alpha(\sigma(N)),
\end{aligned}
$$

i.e., Theorem 2(ii) with $\delta_{2}(N)=\alpha(\sigma(N))$. Thus, Theorem 2 applies and (7) follows with $\varepsilon(N)=\delta_{1}(N)+\delta_{2}(N)$.

With the help of this corollary we can now explain why the receding horizon controller exhibits approximately optimal trajectories in Example 1.

Example 4: We reconsider Example 1 for state constraint set $\mathbb{X}=[-a, a]$ with arbitrary $a \in(0,1]$ and show that the assumptions of Corollary 3 are satisfied. Clearly, $x^{*}=0$ is an equilibrium for $u^{*}=0$ and $\ell_{0}:=0=\ell\left(x^{*}, u^{*}\right) \leq$ $\ell(x, u)$ for all $x \in \mathbb{X}, u \in U$. Using the control sequence $u_{x}(0)=-2 x$ and $u_{x}(k)=0$ for $k \geq 1$ the corresponding trajectory satisfies $x_{u_{x}}(k, x)=x^{*}$ for all $k \geq 1$ and $\ell\left(x_{u_{x}}(k, x), u_{x}(k)\right) \leq(2 x)^{2}$. This proves Assumption (a) of Corollary 3 for $\alpha(r)=4 r^{2}$ and $R=1$.

For checking Assumption (b) of Corollary 3, we use $N_{0}=$ 2 and define $\delta(N):=a / 2^{N-1}$ and $\gamma(N):=a^{2} /\left(N 2^{2 N-1}\right)$. Consider a trajectory satisfying $x_{u}(k, x) \in \mathbb{X}$ and $\mid x_{u}(k, x)$ $x^{*} \mid \geq \delta(N)$, i.e., $\left|x_{u}(k, x)\right| \in[\delta(N), a]$ for all $k=0, \ldots, N$. We first show the inequality

$$
J_{N}(x, u) \geq 2 \gamma(N) .
$$

To this end, by symmetry of the problem we can assume without loss of generality that $x_{u}(N-1, x)>0$. In case that $x \leq \delta(N)$ there must be $k \in\{0, \ldots, N-1\}$ such that $x_{u}(k, x) \leq-\delta(N)$ and $x_{u}(k+1, x) \geq \delta(N)$ implying $u(k) \geq 3 \delta(N)$. This yields $J_{N}(x, u) \geq u(k)^{2} / N \geq$ $9 \delta(N)^{2} / N=9 a^{2} /\left(N 2^{2 N-2}\right) \geq 2 \gamma(N)$ and thus (13). In case $x \geq \delta(N)$ we observe that

$$
a \geq x_{u}(k, x)=\sum_{n=0}^{k-1} 2^{k-n-1} u(n)+2^{k} x .
$$

Hence, for $k=N$ we get

$$
\sum_{n=0}^{N-1} 2^{N-n-1} u(n) \leq a-2^{N} x \leq a-2^{N} \delta(N) \leq-a
$$

implying $u(k) \leq-a / 2^{N-1}$ for some $k \in\{0, \ldots, N-1\}$. This yields $J_{N}(x, u) \geq u(k)^{2} / N \geq a^{2} /\left(N 2^{N-2}\right) \geq 2 \gamma(N)$ and thus again (13).

For $N>N^{\prime}$ we let $i \in \mathbb{N}$ be maximal with $N \geq i N^{\prime}$ which implies $(i+1) N^{\prime} \geq N$ and thus $i N^{\prime} / N \geq i /(i+1) \geq$ $1 / 2$. From $\ell \geq 0$ we get the inequality

$$
J_{N}(x, u) \geq \sum_{j=0}^{i-1} \frac{N^{\prime}}{N} J_{N^{\prime}}\left(x_{u}\left(j N^{\prime}, x\right), u\left(j N^{\prime}+\cdot\right)\right) .
$$

Using (13) with $N^{\prime}=N$ we can then estimate $J_{N^{\prime}}\left(x_{u}\left(j N^{\prime}, x\right), u\left(j N^{\prime}+\cdot\right)\right) \geq 2 \gamma\left(N^{\prime}\right)$ which implies

$$
J_{N}(x, u) \geq i \frac{N^{\prime}}{N} 2 \gamma\left(N^{\prime}\right) \geq \gamma\left(N^{\prime}\right) .
$$

This proves Assumption (b) of Corollary 3.

This construction also explains why $J_{\infty}\left(x, \mu_{N}\right)$ increases when $a$ in $\mathbb{X}=[-a, a]$ increases: the parameter $a$ appears linearly in $\sigma(N)$, because $\delta(N)>\gamma(N)$ and thus $\delta(\eta(N))$ is dominant in the definition of $\sigma(N)$. Since $\alpha(r)=4 r^{2}$, the parameter appears as $a^{2}$ in $\varepsilon(N)$, hence $\varepsilon(N)$ increases with 
increasing $a$. Moreover, the term $a^{2}$ suggests that the values for $\mathbb{X}=[-1,1]$ should be four times as large than those for $\mathbb{X}=[-0.5,0.5]$. This is exactly the case for our numerical results shown in Figure 2.

Remark 5: Corollary 3 does not guarantee that the closed loop solutions converge to a neighborhood of $x^{*}$. Condition (b) and the fact that $J_{K}\left(x_{\mu_{N}}(k), \mu_{N}\right)$ is small for all $k$ and all sufficiently large $N$ and $K$ (which follows from (4)) only ensure that there exist arbitrary large $n$ for which $x_{\mu_{N}}(n)$ is close to $x^{*}$. Currently, it is an open question how to prove the convergence observed numerically in Figure 2. Note that the strong duality condition from [4] is not satisfied for Example 1 , hence we cannot use the arguments from this reference.

\section{AN EXAMPLE OF AN OPTIMAL PERIODIC SOLUTION}

Theorem 2 is not restricted to the case of optimal equilibria. Even if we strengthen condition (ii) of the theorem to convergence $\ell\left(x_{u_{N, x}}(N, x), u_{N, x}(N)\right) \rightarrow \ell_{0}$ as $N \rightarrow \infty$ (which is what we get from Corollary 3), this does not necessarily mean that $x_{u_{N, x}}(N, x)$ must converge as $N \rightarrow$ $\infty$. Thus, we can expect that the receding horizon controller is able to approximate an optimal periodic trajectory, at least when the running cost along this trajectory is constant to $\ell_{0}$. The following example shows that this is indeed the case.

Example 6: Consider the two dimensional control system with $x=\left(x_{1}, x_{2}\right)^{T} \in \mathbb{R}^{2}$ and $u=\left(u_{1}, u_{2}\right)^{T} \in \mathbb{R}^{2}$ given by

$$
x(k+1)=A\left(u_{2}(k)\right)\left(2 x(k)+u_{1}(k) x(k) /\|x(k)\|\right),
$$

for $x(k) \neq 0$ and $x(k+1)=0$ for $x(k)=0$, where

$$
A\left(u_{2}\right)=\left(\begin{array}{rr}
\cos u_{2} & \sin u_{2} \\
-\sin u_{2} & \cos u_{2}
\end{array}\right) \in \mathbb{R}^{2 \times 2}
$$

and $\|\cdot\|$ is the Euclidean norm. We choose the admissible set as the ring $\mathbb{X}=\left\{x \in \mathbb{R}^{2} \mid 3 / 4 \leq\|x\| \leq 2\right\}$, the control value set as $U=[-5,5] \times[-1,1]$ and the stage cost as $\ell(x, u)=\left(u_{1}+1\right)^{2}+\left(u_{2}-0.1\right)^{2}$. With this cost function, one easily sees that it is optimal to first steer the system to the circle $S=\left\{x \in \mathbb{R}^{2} \mid\|x\|=1\right\}$ and then use the control $u^{*}=(-1,0.1)^{T}$. Indeed, since $f\left(x, u^{*}\right) \in S$ and $\ell\left(x, u^{*}\right)=0$ for all $x \in \mathbb{X}$, using $u^{*}$ we stay on $S$ with stage cost 0 and thus for any control sequence $u_{x}$ which first steers the system from $x \in \mathbb{X}$ to $S$ in finitely many steps and then uses the control $u_{x}(k)=u^{*}$ we get $J_{\infty}\left(x, u_{x}\right)=0$. Since $\ell \geq 0$, this is obviously the optimal value. Since $u_{2}^{*}=0.1$ and thus $A\left(u_{2}^{*}\right) \neq \mathrm{Id}$, the corresponding optimal trajectory is not an equilibrium but a periodic orbit.

Figure 4 shows the resulting receding horizon closed loop trajectories for $N=4,6,8$ and initial values $x_{0}=(0,2)^{T}$ (outer trajectories) and $x_{0}=(0,3 / 4)^{T}$ (inner trajectories), respectively. The corresponding averaged infinite horizon closed loop costs are $J_{\infty}\left(x_{0}, \mu_{4}\right)=0.35, J_{\infty}\left(x_{0}, \mu_{6}\right)=$ 0.0022 and $J_{\infty}\left(x_{0}, \mu_{8}\right)=0.00014$ for $x_{0}=(0,2)^{T}$ and $J_{\infty}\left(x_{0}, \mu_{4}\right)=0.0022, J_{\infty}\left(x_{0}, \mu_{6}\right)=0.00014$ and $J_{\infty}\left(x_{0}, \mu_{8}\right)=0.0000086$ for $x_{0}=(0,3 / 4)^{T}$.

As we see, the resulting limit cycle depends on the initial value and its radius is $>1$ for $x_{0}=(0,2)^{T},<1$ for $x_{0}=(0,3 / 4)^{T}$ and converges to 1 in both cases for

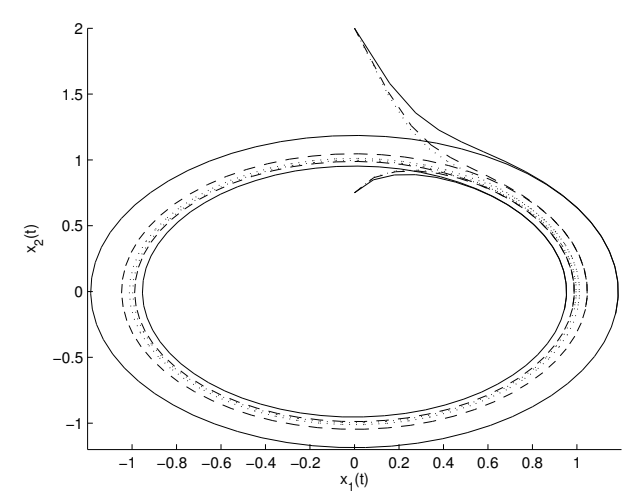

Fig. 4. $x_{\mu_{N}}(k, x)$ for $N=4$ (solid), $N=6$ dashed and $N=8$ (dotted) for $x_{0}=(0,2)^{T}$ (outer trajectories) and $x_{0}=(0,3 / 4)^{T}$ (inner trajectories)

increasing $N$. Furthermore, in both cases for increasing $N$ the solutions improve and the infinite horizon closed loop costs approach the optimal value $V_{\infty}\left(x_{0}\right)=0$. A formal proof of the convergence of the cost could be achieved by an extension of Corollary 3 to the periodic case followed by an analysis similar to Example 4 which is quite straighforward but is omitted here due to space limitations.

It is also interesting to look at the open loop predictions for the different initial values which are depicted in Figure 5 for $N=4$ and $x_{0}=(0,2)^{T}$ and $x_{0}=(0,3 / 4)$, respectively. As in Figure 3, the optimal open loop solutions approach the boundary of the admissible set $\mathbb{X}$ but now it depends on the initial value whether the "outer" boundary $\|x\|=2$ or the "inner" boundary $\|x\|=3 / 4$ is approached.

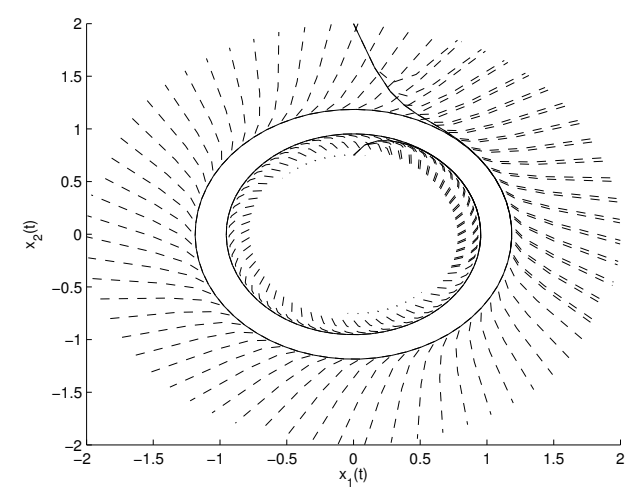

Fig. 5. Optimal predictions $x_{u}\left(k, x_{\mu_{N}}(k)\right)$ (dashed) within the receding horizon optimization for $N=4$ with $x_{0}=(0,2)^{T}$ (outer trajectories) and $x_{0}=(0,3 / 4)^{T}$ (inner trajectories)

\section{CONCLUSIONS AND OUTLOOK}

We have derived conditions under which a receding horizon control scheme yields approximately optimal infinite horizon averaged performance for the resulting closed loop trajectories. The results show that such behavior can be obtained without positive definiteness or detectability assumptions and without imposing terminal constraints and 
incorporating a priori information about the optimal solution in the scheme.

Future research will include the investigation of conditions under which the (approximate) convergence of the closed loop solution to the optimal solution can be shown and the extension to periodic orbits along which $\ell$ is not necessarily constant.

\section{REFERENCES}

[1] D. Angeli, R. Amrit, and J. B. Rawlings. Receding horizon cost optimization for overly constrained nonlinear plants. In Proceedings of the 48th IEEE Conference on Decision and Control - CDC 2009, pages 7972-7977, Shanghai, China, 2009.

[2] D. Angeli and J. B. Rawlings. Receding horizon cost optimization and control for nonlinear plants. In Proceedings of the 8th IFAC Symposium on Nonlinear Control Systems - NOLCOS 2010, pages 1217-1223, Bologna, Italy, 2010.

[3] D. P. Bertsekas. Dynamic Programming and Optimal Control. Vol. 1 and 2. Athena Scientific, Belmont, MA, 1995.

[4] M. Diehl, R. Amrit, and J. B. Rawlings. A Lyapunov function for economic optimizing model predictive control. IEEE Trans. Autom. Control, 2011. To appear.

[5] G. Grimm, M. J. Messina, S. E. Tuna, and A. R. Teel. Model predictive control: for want of a local control Lyapunov function, all is not lost. IEEE Trans. Automat. Control, 50(5):546-558, 2005.

[6] L. Grüne. Analysis and design of unconstrained nonlinear MPC schemes for finite and infinite dimensional systems. SIAM J. Control Optim., 48:1206-1228, 2009.

[7] L. Grüne and J. Pannek. Nonlinear Model Predictive Control. Theory and Algorithms. Springer-Verlag, London, 2011. To appear.

[8] L. Grüne, J. Pannek, M. Seehafer, and K. Worthmann. Analysis of unconstrained nonlinear MPC schemes with varying control horizon. SIAM J. Control Optim., 48:4938-4962, 2010.

[9] D. Q. Mayne, J. B. Rawlings, C. V. Rao, and P. O. M. Scokaert. Constrained model predictive control: stability and optimality. Automatica, 36:789-814, 2000

[10] J. A. Primbs and V. Nevistić. Feasibility and stability of constrained finite receding horizon control. Automatica, 36(7):965-971, 2000.

[11] J. B. Rawlings and D. Q. Mayne. Model Predictive Control: Theory and Design. Nob Hill Publishing, Madison, 2009. 